Nota / Note

\title{
TESTES DE CAPACIDADE DE EXPANSÃO EM PROGRAMAS DE MELHORAMENTO DE MILHO PIPOCA
}

\author{
Frederico de Pina Matta ${ }^{1,3}$; José Marcelo Soriano Viana²* \\ ${ }^{1}$ Pós-Graduando do Depto. de Biologia Geral - UFV. \\ ${ }^{2}$ Depto. de Biologia Geral - UFV - CEP: 36571-000 - Viçosa, MG. \\ ${ }^{3}$ Bolsista CAPES. \\ *Autor correspondente <jmsviana@mail.ufv.br>
}

\begin{abstract}
RESUMO: No desenvolvimento de populações melhoradas de milho pipoca é fundamental selecionar com base em qualidade, normalmente expressa pela relação volume de pipoca/peso de grãos, denominada capacidade de expansão (CE). Na seleção de indivíduos e progênies diversos sistemas de avaliação de qualidade devem ser considerados, por serem utilizados pelos consumidores. O propósito deste trabalho foi avaliar dois sistemas de determinação de CE em programas de melhoramento, sendo o primeiro uma pipoqueira de ar quente e o segundo um forno de microondas. Para avaliar a pipoqueira de ar quente foi instalado um experimento no delineamento inteiramente casualizado, envolvendo três tratamentos e nove níveis de peso de grãos, com cinco repetições. Para o microondas, buscando definir uma embalagem alternativa, foi instalado um experimento no delineamento inteiramente casualizado, seguindo um esquema fatorial, com três repetições, envolvendo dois tempos e cinco embalagens. Foi realizado um outro experimento, buscando verificar a qualidade do aparelho para obtenção de CE a partir de pequena quantidade de grãos e qual o melhor tempo a ser utilizado. A pipoqueira de ar quente é um sistema eficiente, podendo ser empregados $10 \mathrm{~g}$ na avaliação de plantas e até $90 \mathrm{~g}$ para avaliações experimentais de famílias. O forno de microondas é também um sistema eficiente, equivalente à pipoqueira de ar quente. No aparelho de microondas, pode-se empregar saco de papel kraft. Para avaliação de plantas, podem ser empregados 10 gramas e 140 segundos, e 30 a 90 gramas com tempo de 220 segundos para a avaliação de progênies.
\end{abstract}

Palavras-chave: pipocadoras, avaliação de qualidade

\section{POPPING EXPANSION TESTS IN POPCORN BREEDING PROGRAMS}

\begin{abstract}
Quality, normally expressed by the relation volume of popcorn/weight of grains (volume expansion), is an important characteristic in popcorn breeding. Different systems of assessing popcorn expansion should be used in individual and family selections, since they are common to consumers. The purpose of this work was to evaluate two systems for popping expansion determination in breeding programs. The first was a hot air popcorn pumper and the second was a microwave oven. For the first one an experiment was installed in a completely randomized design, with three treatments and nine levels of kernel weight, and five replications. For the second one, aiming to define an alternative packaging, an experiment was installed also in a completely randomized design, following a factorial scheme, with three replications, involving two times and five packagings. Another experiment was achieved to verify the microwave oven quality for volume expansion determination with a little kernel quantity and the best time to be used. The hot air popcorn pumper is an efficient system, in which $10 \mathrm{~g}$ of kernel can be used to evaluate plants and up to $90 \mathrm{~g}$ of kernel can be used to evaluate families in the experiments. The microwave oven is equivalent to the hot air popcorn pumper. Kraft paper bags can be used in the microwave oven. For plant evaluation $10 \mathrm{~g}$ of kernel with 140 seconds are recommended. To evaluate progenies $30 \mathrm{~g}$ to $90 \mathrm{~g}$ of kernel with 220 seconds can be used.

Key words:quality
\end{abstract}

\section{INTRODUÇÃO}

A cultura do milho pipoca no Brasil vem recebendo ultimamente maior atenção pelos melhoristas e produtores, devido ao aumento de consumo, principalmente em razão da chegada de milho pipoca importado dos Estados Unidos, para uso em fornos de microondas. Como o mercado de produtos agrícolas é um fator determinante nos rumos da agricultura, seu cultivo tende a expandir-se ainda mais em razão desse crescente consumo. O valor do milho pipoca depende principalmente de sua qualidade, medida pela capacidade de expansão (CE), pela maciez e pelo sabor (Brunson, 1931). A CE corresponde à relação entre o volume de pipoca e o volume ou o peso de grãos. Green \& Harris (1960) descrevem que a capacidade de expansão é afetada principalmente pelo teor de umidade dos grãos, mas, também, por outros fatores, como grau de dano no pericarpo e endosperma e pelo método de secagem. Os autores recomendam avaliar CE quando o teor de umidade está entre 13 e $14 \%$. 
Com base no Projeto de Normas de Identidade e Qualidade de Milho Pipoca, do Ministério da Agricultura e Abastecimento, ainda não aprovado, para comercialização uma população precisa ter CE de no mínimo $15 \mathrm{~mL} \mathrm{~mL}^{-1}$ (Pacheco et al., 1996). Segundo Green \& Harris (1960), população com CE menor que $25 \mathrm{~mL} \mathrm{~g}^{-1}$ é considerada pobre. Se a CE está entre $25 \mathrm{e}$ 30 ela é considerada como regular. Valores entre 30 e 35 são considerados como bons e populações com CE acima de 35 são classificadas como excelentes. Além disso, citam que os sistemas de avaliação 'Official Volume Tester' (volume por volume), usado entre 1916 e 1956, e 'Weight Volume Tester' (volume por peso), adotado a partir de 1956, são equivalentes.

Nos programas de melhoramento intrapopulacional com seleção dentro e de produção de linhagens, é necessário avaliar a CE de plantas na seleção em lote de recombinação e em progênies endogâmicas. Esta medida deve ser feita a partir de uma reduzida quantidade de grãos, de forma a garantir adequada disponibilidade de sementes remanescentes.

A crescente utilização pelo consumidor de aparelho de microondas, devido a sua praticidade e possibilidade de uso para vários tipos de alimentos, tem sido verificada pela quantidade de novos produtos desenvolvidos, como o milho pipoca de microondas. Desde que Dofing et al. (1990) relataram uma interação significativa entre genótipo e pipoqueira, usando um aparelho de microondas e outros convencionais para o pipocamento, os programas de melhoramento vem se utilizando de vários métodos, com o objetivo de melhor caracterização dos genótipos, de acordo com sua finalidade de processamento.

O propósito deste trabalho foi avaliar dois sistemas de determinação de CE em programas de melhoramento, sendo o primeiro uma pipoqueira de ar quente e o segundo um forno de microondas.

\section{MATERIAL E MÉTODOS}

\section{Pipoqueira de ar quente}

Foi instalado um experimento no delineamento inteiramente casualizado, envolvendo três tratamentos de milho pipoca e nove níveis de peso de grãos, com cinco repetições. Os tratamentos utilizados foram duas marcas comerciais (marcas 1 e 2) e a população Viçosa, sob melhoramento no Programa de Melhoramento de Milho Pipoca do Setor de Genética do Departamento de Biologia Geral, da UFV. Foram retiradas amostras de 10 , $20,30,40,50,60,70,80$ e $90 \mathrm{~g}$ de grãos de cada tratamento, após determinação do teor de umidade.

$\mathrm{Na}$ determinação de CE foi utilizada a pipoqueira de ar quente Hot Air Popcorn Pumper H7340, da Proctor Silex, com 1250 watts de potência, cuja capacidade máxima de grãos é $90 \mathrm{~g}$. O sistema empregado foi o mesmo adotado por Matta \& Viana (1996), o qual consiste em colocar os grãos no recipiente da pipoqueira quando a temperatura atingir $100^{\circ} \mathrm{C}$. Esse processo de pré-aquecimento é muito recomendado pelos melhoristas de milho pipoca, com o objetivo de se reduzir a variação existente entre as mensurações (Roshdy et al., 1984; Metzger et al., 1989; Song et al., 1991). Na mensuração do volume de pipoca foi utilizada uma proveta de 1000 $\mathrm{ml}$, com funil. O peso de grãos não estourados (piruás) também foi mensurado.

\section{Forno de microondas}

O aparelho utilizado foi um forno de microondas Electrolux ME 900 com capacidade de 30 litros e potência de 900 watts. Visando verificar seu desempenho utilizando milho para microondas e, portanto, em embalagem própria, foram utilizadas duas versões da marca comercial 2, uma sem sal e sabor e outra com sal e sabor e dois níveis de tempo, o recomendado pelo fabricante (190 segundos) e o tempo de 200 segundos. $O$ delineamento empregado foi $O$ inteiramente casualizado, com duas repetições. A CE foi calculada considerando $80 \mathrm{~g}$, que é o peso médio de grãos limpos por embalagem.

Buscando definir uma embalagem que possa ser empregada em programas de melhoramento, em lugar da embalagem desenvolvida para este fim, sem a gordura vegetal, foi instalado um experimento no delineamento inteiramente casualizado, seguindo um esquema fatorial, com três repetições, envolvendo dois tempos e cinco embalagens. As embalagens foram duas pipoqueiras plásticas (embalagens 1 e 2), pacote de papel comum pardo (embalagem 3), a embalagem própria limpa, após serem removidos os grãos embebidos com gordura vegetal (embalagem 4), e essa mesma embalagem, também limpa, após o uso no microondas e retiradas as pipocas (embalagem 5 ). Os tempos foram o recomendado pelo fabricante do aparelho de microondas, que são 190 segundos, sob potência máxima, e o tempo de quatro minutos, também sob potência máxima, indicado pelo fabricante de uma das pipoqueiras plásticas.

Para cada tratamento foi utilizada uma amostra de 80 gramas de grãos da marca comercial 2 para microondas, sem sabor e sem sal. Para obtenção desses grãos, foi necessário retirá-los dos pacotes comerciais e limpá-los, removendo-se toda a gordura vegetal existente entre os grãos.

Tendo determinado a melhor embalagem, foi realizado um terceiro experimento, buscando verificar a qualidade do aparelho para obtenção de CE a partir de pequena quantidade de grãos e qual o melhor tempo a ser utilizado. Esse experimento foi instalado em um esquema fatorial, com três repetições, envolvendo duas populações de milho pipoca, dois níveis de peso de grãos e quatro tempos sob potência máxima. Os tratamentos utilizados foram a marca comercial 2 e a população Viçosa. Os pesos utilizados foram de 30 e 90 g. Esses pesos foram utilizados por serem o usado pelo 
programa de melhoramento de milho pipoca da UFV e por ser a quantidade recomendada pela pipoqueira de ar quente, respectivamente.

Os tempos foram o recomendado pelo fabricante do aparelho, ou seja, 190 segundos, e os tempos 200, 210 e 220 segundos, todos sob potência máxima. Neste experimento, dois tratamentos foram adicionados: a marca comercial $2 \mathrm{com} 10$ gramas de grãos e 140 segundos e a população Viçosa com 10 gramas de grãos e 140 segundos. O tempo de 140 segundos foi utilizado por ser o máximo observado previamente ao experimento, antes do início da queima da pipoca para essa quantidade de grãos.

O método utilizado para a mensuração do volume de pipoca foi o mesmo descrito para a pipoqueira de ar quente. Antes da determinação de CE, foi mensurado o teor de umidade dos grãos. A quantidade de piruás também foi anotada.

\section{RESULTADOS E DISCUSSÃO}

\section{Pipoqueira de ar quente}

De acordo com as análises de variância de CE e Peso de Piruás (PI), a interação entre populações e pesos de grãos foi estatisticamente significativa a $5 \%$. A população Viçosa apresentou média de $13,1 \%$ de umidade e as marcas comerciais 1 e 2 apresentaram $12,8 \%$ e $14,4 \%$, respectivamente. Esses valores, de acordo com Lyerly (1940) e Ziegler et al. (1985), citados por Metzger et al. (1989), estão dentro do intervalo recomendado para uma melhor determinação de CE.

Para a característica CE, levando em consideração os níveis do fator população dentro de cada nível do fator peso de grãos (TABELA 1), verificase que apenas para os níveis de 10 e $20 \mathrm{~g}$, as duas marcas comerciais não diferiram entre si. Vale salientar que Viçosa, uma população de polinização aberta sob melhoramento, apresenta uma CE média bem superior ao mínimo estipulado para comercialização, $24,3 \mathrm{~mL} \mathrm{~g}^{-1}$, além de apresentar uma produtividade em torno de $2500 \mathrm{~kg} \mathrm{ha}^{-1}$, superior à média geral obtida no Ensaio Nacional de Milho Pipoca, no ano agrícola 91/92, que foi de $2075 \mathrm{~kg} \mathrm{ha}^{-1}$, no qual o cultivar mais produtivo produziu $3058 \mathrm{~kg} \mathrm{ha}^{-1}$ (Andrade, 1996).

$\mathrm{Na}$ maioria dos casos a marca comercial 2 foi a que apresentou maior quantidade de piruás, enquanto que a população Viçosa foi a que menos produziu piruás. Para os pesos 60, 80 e $90 \mathrm{~g}$, houve uma alteração nas posições entre os tratamentos. Entretanto os valores apresentados para esses níveis de peso são uniformes entre si.

Analisando os níveis de peso de grãos dentro de cada população, foi verificada relação funcional entre peso de grãos e CE somente para as marcas comerciais 1 e 2 . Em relação à marca comercial 2, observa-se existência de relação funcional quadrática, cuja função é $Y=31,4217+0,290837 X-0,002045 X^{2}$, com r $^{2}=0,85$ (Figura 1), indicando que a CE máxima seria obtida com o uso de $71,12 \mathrm{~g}$ de grãos. Para $\mathrm{PI}$, com função $\mathrm{Y}=$ 2,50556 - 0,008333X, há uma tendência de redução na quantidade de piruás com o aumento da quantidade de grãos utilizados. Assim, observa-se uma diminuição na quantidade de piruás com o aumento no peso de grãos, paralelamente a um acréscimo na CE.

Quanto à marca 1, observa-se a existência de uma relação funcional linear entre CE e peso de grãos, cuja função é $Y=31,7141+0,049163 X$, com $r^{2}=0,84$ (Figura 2). Quanto maior o peso de grãos utilizados, maior será a $\mathrm{CE}$, entre os níveis de peso utilizados neste trabalho. Para PI, a função $Y=1,5333+0,0092 X$ indica uma tendência de aumento na quantidade de piruás com o aumento da quantidade de grãos utilizados.

Para a população Viçosa não foi verificada relação funcional entre CE e peso de grãos, ou seja, o valor de CE é uma constante para todo peso (Figura 3). $A$ ausência de relação funcional entre CE e peso de grãos informa que em programas de melhoramento populacional e de obtenção de linhagens, o uso de $10 \mathrm{~g}$ já seria suficiente para caracterização da qualidade das unidades de seleção, plantas e famílias.

Embora as marcas comerciais não sejam populações sob o ponto de vista genético, os resultados das regressões evidenciam que em populações de elevada CE há relação funcional entre CE e peso de grãos. Portanto, na caracterização da qualidade de material superior, é necessário utilizar o máximo de grãos permitidos $(90 \mathrm{~g})$.

Para PI, a função $Y=0,656111+0,0155 X$ revela uma tendência de aumento na quantidade de piruás com o aumento da quantidade de grãos utilizados. Dessa

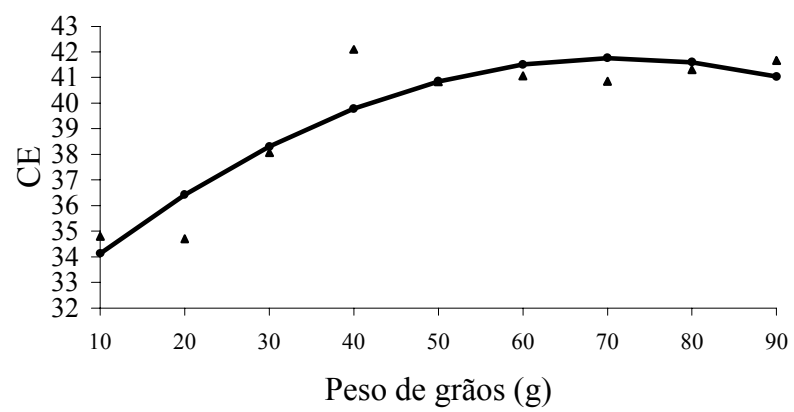

Figura 1 - Relação entre capacidade de expansão (CE) e peso de grãos de milho pipoca, utilizando a marca comercial 2 em pipoqueira de ar quente.

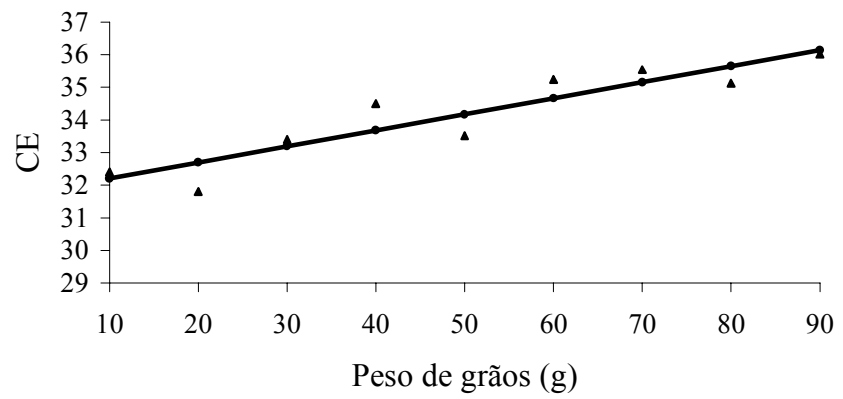

Figura 2 - Relação entre capacidade de expansão (CE) e peso de grãos de milho pipoca, utilizando a marca comercial 1em pipoqueira de ar quente. 
TABELA 1 - Médias de Capacidade de Expansão (CE) e de Peso de Piruás (PI) das Marcas Comerciais 1 e 2 e da População Viçosa, em Cada Nível do Fator Peso, em Pipoqueira de Ar Quente ${ }^{1 /}$.

\begin{tabular}{|c|c|c|c|c|c|c|}
\hline \multirow{2}{*}{ Peso } & \multicolumn{3}{|c|}{ CE } & \multicolumn{3}{|c|}{ PI } \\
\hline & Marca 2 & Marca 1 & Viçosa & Marca 2 & Marca 1 & Viçosa \\
\hline$g$ & 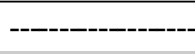 & $-\mathrm{mL} \mathrm{g}^{-1}$ & --.---- & -------------' & --- g ----י-- & ---------_. \\
\hline 10 & $34,8 \mathrm{a}$ & $32,4 \mathrm{a}$ & $24,2 \mathrm{~b}$ & $2,14 \mathrm{a}$ & $1,54 a b$ & $0,92 b$ \\
\hline 20 & $34,7 \mathrm{a}$ & $31,8 \mathrm{a}$ & $25,2 \mathrm{~b}$ & $2,86 \mathrm{a}$ & $1,98 a b$ & $1,08 b$ \\
\hline 30 & $38,1 \mathrm{a}$ & $33,4 \mathrm{~b}$ & $24,5 \mathrm{c}$ & $1,90 \mathrm{a}$ & $1,72 \mathrm{a}$ & $1,08 \mathrm{a}$ \\
\hline 40 & $42,1 \mathrm{a}$ & $34,5 \mathrm{~b}$ & $23,4 \mathrm{c}$ & $2,00 \mathrm{a}$ & $1,82 a$ & $1,18 \mathrm{a}$ \\
\hline 50 & $40,8 \mathrm{a}$ & $33,5 b$ & $24,1 \mathrm{c}$ & $2,54 \mathrm{a}$ & $1,76 a b$ & $1,14 \mathrm{~b}$ \\
\hline 60 & $41,1 \mathrm{a}$ & $35,2 \mathrm{~b}$ & $23,7 \mathrm{c}$ & $1,92 \mathrm{a}$ & $2,40 a$ & $1,76 \mathrm{a}$ \\
\hline 70 & $40,8 \mathrm{a}$ & $35,5 \mathrm{~b}$ & $24,9 \mathrm{c}$ & $2,14 \mathrm{a}$ & $2,10 a$ & $1,56 \mathrm{a}$ \\
\hline 80 & $41,3 a$ & $35,1 \mathrm{~b}$ & $24,2 \mathrm{c}$ & $1,46 a$ & $2,20 \mathrm{a}$ & $1,96 \mathrm{a}$ \\
\hline 90 & $41,7 \mathrm{a}$ & $36,0 \mathrm{~b}$ & $24,5 \mathrm{c}$ & $1,84 \mathrm{a}$ & $2,42 a$ & $2,20 a$ \\
\hline
\end{tabular}

${ }^{1 / A s}$ médias seguidas pela mesma letra, para cada característica, em cada linha, não diferem a $5 \%$ pelo teste de Tukey.

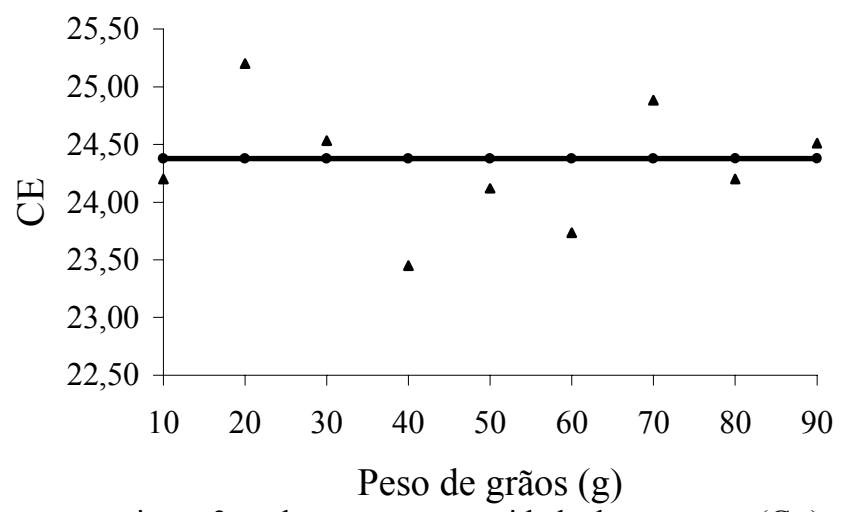

Figura 3 - Relação entre capacidade de expansão (CE) e peso de grãos, utilizando a população Viçosa em pipoqueira de ar quente.

forma, para populações sob melhoramento, ao trabalhar com plantas justifica-se o uso de apenas $10 \mathrm{~g}$ de grãos, pois além de fornecer estatisticamente a mesma estimativa de CE, haverá economia de sementes.

$\mathrm{Na}$ avaliação de famílias pode-se empregar até $90 \mathrm{~g}$, embora a este peso esteja associada a maior quantidade de piruás. No programa de melhoramento de milho pipoca do Setor de Genética da Universidade Federal de Viçosa, são utilizados $10 \mathrm{~g}$ na avaliação de plantas e $30 \mathrm{~g}$ na avaliação de famílias.

\section{Forno de microondas}

Não foi verificado efeito dos condimentos na característica CE e na quantidade de piruás. Esse resultado não concorda com o obtido por Mohamed et al. (1993), em que o uso de sal diminui a CE. O acréscimo de dez segundos também não acarretou em alguma mudança significativa nestas duas variáveis.

De acordo com as análises de variância de CE e PI, para o experimento envolvendo cinco embalagens e dois níveis de tempo sob potência máxima, verificouse que a interação entre os fatores não foi estatisticamente significativa a 5\%. Há, contudo, diferenças entre as embalagens e entre os tempos.
As embalagens 1 e 2, que são potes plásticos comerciais, não apresentaram um resultado satisfatório, sendo inferiores às demais (TABELA 2). Como era de se esperar, as embalagens 4 e 5 , por terem sido desenvolvidos para essa finalidade, apresentaram resultados superiores, enquanto que o resultado da embalagem 3 foi de certa forma surpreendente e muito satisfatório, pois comparativamente às embalagens planejadas para este fim, nova e usada, suas vantagens são a fácil obtenção e seu baixíssimo custo.

Verifica-se ainda a superioridade das médias de CE para o nível de 240 segundos sobre o tempo recomendado pelo fabricante do aparelho (TABELA 2). Entretanto, mesmo reduzindo a quantidade de piruás, este maior tempo foi prejudicial para a qualidade da pipoca, apresentando uma pequena quantidade de pipocas queimadas na maioria das embalagens. A embalagem 4 com o tempo de quatro minutos foi a que apresentou maior quantidade de pipocas queimadas.

As embalagens 1 e 2 foram as que deixaram maior quantidade de piruás. O resultado da embalagem 3 foi novamente surpreendente, demonstrando ser eficiente a avaliação de CE com esse material, por deixar também pouca quantidade de piruás.

Comparando-se os resultados obtidos no experimento anterior, com o tempo de 190 segundos, sem limpar os grãos, com os obtidos neste, verifica-se que a gordura vegetal desempenha papel importante no aumento da capacidade de expansão. Ela proporcionou um aumento médio de 30,42 para 40,31, além do já relatado na literatura, aumento da maciez da pipoca e do melhor paladar.

Considerando os resultados da pipoqueira de ar quente e do aparelho de microondas, em relação aos dados de CE obtidos com o uso de $80 \mathrm{~g}$ da marca comercial 2, verifica-se que as médias foram estatisticamente iguais (TABELA 3). Vale ressaltar que 
TABELA 2 - Médias de Capacidade de Expansão (CE) e Peso de Piruás (PI) Para as Combinações das Cinco Embalagens Com os Dois Tempos".

\begin{tabular}{ccccc}
\hline \multirow{2}{*}{ Embalagem } & \multicolumn{3}{c}{ CE } & \multicolumn{2}{c}{ PI } \\
\cline { 2 - 5 } & \multicolumn{1}{c}{$190 \mathrm{~s}$} & $240 \mathrm{~s}$ & $190 \mathrm{~s}$ & $240 \mathrm{~s}$ \\
\hline 1 & $19,38 \mathrm{~b} \mathrm{~B}$ & $26,13 \mathrm{~b} \mathrm{~A}$ & 40,33 a A & 26,00 a B \\
\hline 2 & $16,67 \mathrm{~b} \mathrm{~B}$ & $26,54 \mathrm{~b} \mathrm{~A}$ & 39,67 a A & 19,43 a b B \\
3 & 30,17 a B & 35,38 a A & 22,00 b A & 8,67 b B \\
4 & 30,42 a A & 31,88 a b A & 17,00 b A & 17,67 a b A \\
5 & 26,67 a A & 28,96 a b A & 26,83 a b A & 21,50 a b A \\
\hline
\end{tabular}

${ }^{1 / A s}$ médias seguidas pela mesma letra minúscula, em cada coluna, não diferem a $5 \%$ pelo teste de Tukey. Para cada característica, as médias seguidas pela mesma letra maiúscula, em cada linha, não diferem a $5 \%$ pelo teste de Tukey. As embalagens 1 e 2 são duas marcas comerciais plásticas, a embalagem 3 é pacote de papel comum pardo, a embalagem 4 é a comercial, limpa após serem removidos os grãos, e a embalagem 5 é a mesma, após uso no microondas, retiradas as pipocas e limpa.

a CE média no aparelho de microondas foi obtida com o uso de embalagem própria e, portanto, os grãos estavam embebidos na gordura vegetal. No experimento utilizando a pipoqueira de ar quente, a CE foi obtida com a versão comum da marca comercial 2, vendida em pacotes de 500 gramas. Para PI, verifica-se que a média obtida na pipoqueira de ar quente foi estatisticamente inferior à obtida no aparelho de microondas.

Após a definição do saco de papel comum como sendo a embalagem alternativa para os trabalhos de melhoramento de milho pipoca, com o uso de aparelho de microondas, foi realizado um fatorial envolvendo quatro níveis de tempo, duas populações e os dois níveis de peso de grãos, com dois tratamentos adicionais.

Para as características CE e PI, a interação tripla foi estatisticamente significativa, indicando uma dependência entre os níveis dos fatores. Verificou-se que a média dos tratamentos adicionais $(33,0)$ foi estatisticamente diferente da média dos demais tratamentos $(26,08)$, o mesmo ocorrendo para $\mathrm{PI}$, cujas médias foram de $1,5 \mathrm{~g}$ e $14,08 \mathrm{~g}$ para os tratamentos adicionais e os demais tratamentos, respectivamente. Dessa forma, o uso de 10 gramas de grãos no aparelho de microondas é satisfatório dentro de um programa de melhoramento, devendo-se entretanto utilizar um tempo menor que o recomendado pelo fabricante.

As médias apresentadas pela população Viçosa, para a característica PI, foram, em geral, as mesmas ou até mesmo inferiores às apresentadas pela marca comercial 2 (TABELA 4).

A marca comercial apresentou uma quantidade média piruás de $26,25 \%$ do peso original de grãos, quando se utilizou o peso de $30 \mathrm{~g}$, enquanto que para $90 \mathrm{~g}$ de grãos a quantidade de piruás representou, em média, $25,97 \%$ do peso original. Para Viçosa, as médias foram de $22,08 \%$ e $20,51 \%$, para os pesos de $30 \mathrm{~g}$ e 90 $\mathrm{g}$, respectivamente. Dessa forma, a quantidade de piruás, dentro de cada população, é, em média, praticamente a mesma, independentemente da quantidade de grãos utilizados.
TABELA 3 - Comparação de Capacidade de Expansão (CE) e Peso de Piruás (PI) Obtidos com uma Pipoqueira de Ar Quente e um Aparelho de Microondas, com Amostras de 80 Gramas de Grãos da Marca Comercial 2"1.

\begin{tabular}{|c|c|c|c|}
\hline \multicolumn{2}{|c|}{ CE } & \multicolumn{2}{|c|}{$\mathrm{PI}$} \\
\hline Ar Quente & Microondas & Ar Quente & Microondas \\
\hline \multicolumn{2}{|c|}{ - } & \multicolumn{2}{|c|}{ 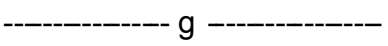 } \\
\hline 41,3 a & 40,31 a & $1,46 \mathrm{~b}$ & $9,13 \mathrm{a}$ \\
\hline
\end{tabular}

${ }^{1 / A s}$ médias foram comparadas pelo teste $\mathrm{t}$ a $5 \%$, considerando amostras com variâncias equivalentes para a característica CE e amostras presumindo variâncias diferentes para a característica peso de piruás. As médias seguidas pela mesma letra, para cada característica, não diferem estatisticamente.

Ao fixarmos peso e tempo, verifica-se que a população Viçosa possui valores de CE muito próximos, e até mesmo equivalentes para alguns tratamentos, da marca comercial 2. Dessa forma, fica claro o potencial de Viçosa para programa de melhoramento intrapopulacional, já que esta é uma população de polinização aberta, sob melhoramento.

Para a marca comercial 2, somente para o tempo de $190 \mathrm{~s}$ foi verificado maior CE com o uso de $30 \mathrm{~g}$ de grãos. Com os tempos de $200 \mathrm{~s}$ e $210 \mathrm{~s}$ os pesos diferentes de grãos proporcionaram CE equivalentes, enquanto que para $220 \mathrm{~s}$ a CE proporcionada com o uso de $90 \mathrm{~g}$ foi superior à apresentada com o peso de $30 \mathrm{~g}$. Para a população Viçosa, verifica-se que, ao fixarmos o tempo, as médias de CE apresentadas foram equivalentes para os pesos utilizados. Assim, para uma avaliação experimental, o uso de $30 \mathrm{~g}$ de grãos já seria suficiente para determinação de CE. Como o uso do tempo de $220 \mathrm{~s}$ proporcionou maior CE, essa combinação seria a ideal para avaliações experimentais em aparelhos de microondas.

Observa-se a existência de relação funcional entre CE e tempo, envolvendo a marca comercial 2 com o uso de 30 gramas, cuja função é $Y=52,9217$ $0,121167 X$, com $r^{2}=0,70$ (Figura 4). 
Assim, com o aumento de tempo, observa-se uma clara redução da CE. Este foi um resultado de certa forma surpreendente, pois se esperava que, com um tempo maior, menor fosse a quantidade de piruás e, dessa forma, maior a CE. Para PI, verifica-se ausência de relação funcional com níveis de tempo, com o uso de $30 \mathrm{~g}$ de grãos. Dessa forma, aumentando o tempo não há estatisticamente uma alteração na quantidade de piruás. Assim, como não ocorre redução de piruás pelo aumento de tempo, é recomendável o uso de 190 segundos por proporcionar maior CE.

Com a marca comercial 2 e com peso de 90 gramas, observa-se uma relação funcional linear, cuja função é $Y=-28,03557+0,276633 X$, com $^{2}=0,89$ (Figura 5). O aumento de tempo proporcionou aumento de CE. A função $Y=156,283-0,648333 X$, existente entre a variável $\mathrm{PI}$ e os níveis de tempo, demonstra que para $90 \mathrm{~g}$ de grãos é recomendável o uso do tempo de 220 segundos, por proporcionar maior CE e menor quantidade de piruás, como já era de se esperar.

Com a população Viçosa e peso de 30 gramas, com função $Y=-7,23934+0,148867 X,{\text { com } r^{2}=0,93}^{2}$ (Figura 6), observa-se aumento de CE com o aumento de tempo. Quanto à $\mathrm{PI}$, verifica-se novamente, para o peso de $30 \mathrm{~g}$, a inexistência de relação funcional com os níveis de tempo. Dessa forma, verifica-se que para

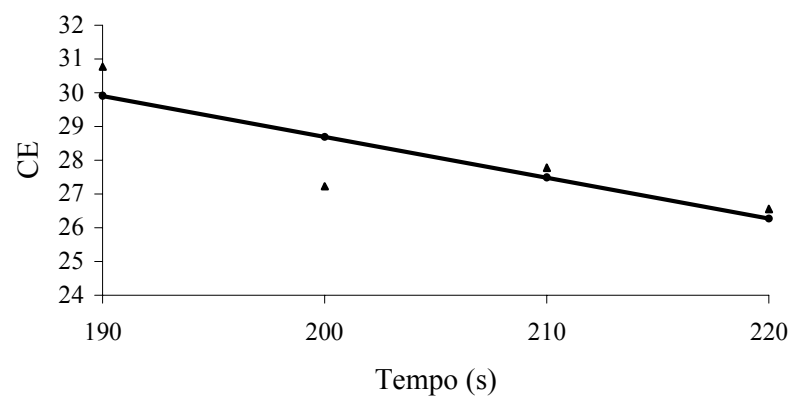

Figura 4 - Relação entre capacidade de expansão (CE) e tempo para a marca 2 , com peso de $30 \mathrm{~g}$, em forno de microondas. esta população é melhor o uso do tempo de 220 segundos, pois proporciona maior $\mathrm{CE}$.

Ainda para a população Viçosa, mas com peso de 90 gramas, a função linear $Y=-7,66+0,155867 X$, com $r^{2}=0,79$ (Figura 7), indica aumento de CE com o aumento de tempo. Para PI, a função $Y=91,2333$ $0,355 \mathrm{X}$ demonstra uma queda da quantidade de piruás com o aumento de tempo. Assim, da mesma forma como para a marca comercial 2, recomenda-se o uso do tempo de 220 segundos para obtenção de uma maior CE com menor quantidade de piruás.

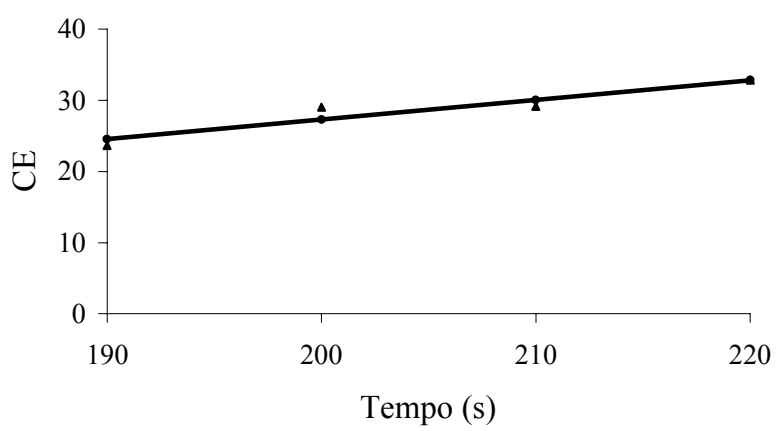

Figura 5 - Relação entre capacidade de expansão (CE) e tempo para a marca 2, com peso de $90 \mathrm{~g}$, em forno de microondas.

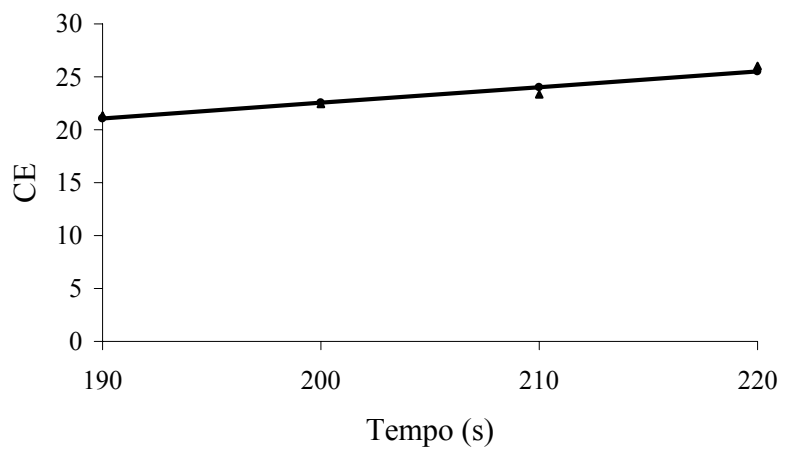

Figura 6 - Relação entre capacidade de expansão (CE) e tempo para a população Viçosa, com peso de $30 \mathrm{~g}$, em forno de microondas.

TABELA 4 - Médias de Capacidade de Expansão (CE) e Peso de Piruás (PI), Após Aquecimento em Forno de Microondas, Obtidos no Fatorial ${ }^{11}$.

\begin{tabular}{lcrrr}
\hline \multirow{2}{*}{ Peso (gramas) e Tempo (segundos) } & \multicolumn{2}{c}{ CE } & \multicolumn{2}{c}{ PI } \\
\cline { 2 - 5 } & Marca 2 & Viçosa & Marca 2 & Viçosa \\
\hline$P=10$ e $T=140$ & $37,00 \mathrm{a}$ & $29,00 \mathrm{~b}$ & $1,67 \mathrm{a}$ & $1,33 \mathrm{a}$ \\
$\mathrm{P}=30$ e $T=190$ & $30,78 \mathrm{a}$ & $21,33 \mathrm{~b} \mid$ & $7,00 \mathrm{a}$ & $9,00 \mathrm{a}$ \\
$\mathrm{P}=90$ e $T=190$ & $23,67 \mathrm{a}$ & $22,19 \mathrm{a} \mid$ & $35,00 \mathrm{a}$ & $23,50 \mathrm{~b}$ \\
$\mathrm{P}=30$ e $T=200$ & $27,22 \mathrm{a} \mid$ & $22,45 \mathrm{~b} \mid$ & $8,33 \mathrm{a}$ & $6,67 \mathrm{a}$ \\
$P=90$ e $T=200$ & $29,03 \mathrm{a} \mid$ & $22,48 \mathrm{~b} \mid$ & $23,50 \mathrm{a}$ & $21,67 \mathrm{a}$ \\
$\mathrm{P}=30$ e $T=210$ & $27,78 \mathrm{a} \mid$ & $23,33 \mathrm{~b} \mid$ & $8,17 \mathrm{a}$ & $6,33 \mathrm{a}$ \\
$P=90$ e $T=210$ & $29,15 \mathrm{a} \mid$ & $26,41 \mathrm{a} \mid$ & $20,67 \mathrm{a}$ & $14,67 \mathrm{~b}$ \\
$P=30$ e $T=220$ & $26,55 \mathrm{a}$ & $26,00 \mathrm{a} \mid$ & $8,00 \mathrm{a}$ & $4,50 \mathrm{a}$ \\
$P=90$ e $T=220$ & $32,85 \mathrm{a}$ & $26,07 \mathrm{~b} \mid$ & $14,33 \mathrm{a}$ & $14,00 \mathrm{a}$ \\
\hline
\end{tabular}

${ }^{1 / A s ~ m e ́ d i a s ~ s e g u i d a s ~ p o r ~ u m a ~ b a r r a, ~ e m ~ c a d a ~ c o l u n a, ~ n a ̃ o ~ d i f e r e m ~ a ~} 5 \%$ pelo teste de Tukey. Para cada característica, as médias seguidas por mesma letra, em cada linha, não diferem a $5 \%$ pelo teste de Tukey. 
Comparando-se a pipoqueira de ar quente com o aparelho de microondas, utilizando a população Viçosa, com pesos de $10 \mathrm{~g}, 30 \mathrm{~g}$ e $90 \mathrm{~g}$ de grãos, verifica-se que apenas para $10 \mathrm{~g}$ os valores de CE foram distintos estatisticamente (TABELA 5).

Para avaliação de CE em programas de melhoramento intrapopulacional com seleção dentro e de produção de linhagens, o uso de $10 \mathrm{~g}$ no aparelho de microondas, com o tempo de $140 \mathrm{~s}$, apresenta valor de CE superior ao apresentado pela pipoqueira de ar quente. Em avaliações de famílias os dois sistemas são estatisticamente equivalentes. Quanto à característica PI, verifica-se que para o peso de $10 \mathrm{~g}$ as médias foram equivalentes, enquanto que para os pesos de $30 \mathrm{~g} \mathrm{e} 90 \mathrm{~g}$ os valores apresentados pelo aparelho de microondas foram estatisticamente maiores.

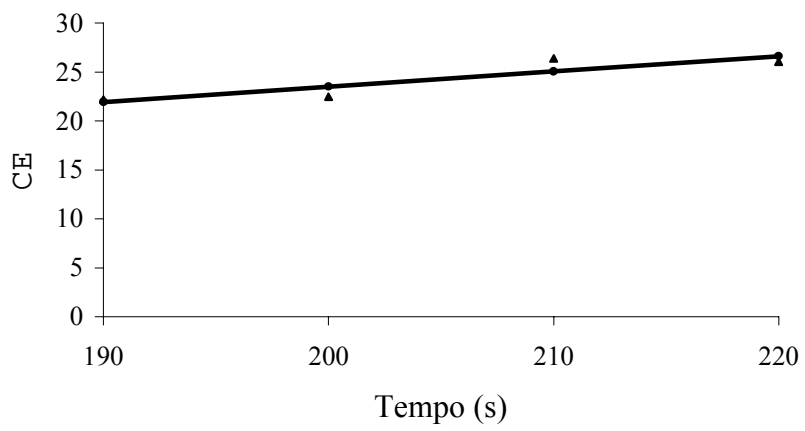

Figura 7 - Relação entre capacidade de expansão (CE) e tempo para a população Viçosa, com peso de 90 gramas, em forno de microondas.

TABELA 5 - Comparação de Capacidade de Expansão (CE, $\mathrm{mL} \mathrm{g}^{-1}$ ) e Peso de Piruás (PI, g) Obtidos com o uso de uma Pipoqueira de Ar Quente e de um aparelho de Microondas, com Amostras de 10, 30 e 90 Gramas de Grãos da População Viçosa ${ }^{1 /}$

\begin{tabular}{ccccc}
\hline Peso & \multicolumn{2}{c}{ CE } & \multicolumn{2}{c}{$\mathrm{Pl}$} \\
\cline { 2 - 5 } & $\begin{array}{c}\mathrm{Ar} \\
\text { Quente }\end{array}$ & Microondas & $\begin{array}{c}\mathrm{Ar} \\
\text { Quente }\end{array}$ & Microondas \\
\hline $\mathrm{g}$ & $------\mathrm{mL} \mathrm{g}^{-1}$-------- & ------ & $\mathrm{g}$ & -------- \\
10 & $24,20 \mathrm{~b}$ & $29,00 \mathrm{a}$ & $0,92 \mathrm{a}$ & $1,33 \mathrm{a}$ \\
30 & $24,53 \mathrm{a}$ & $26,00 \mathrm{a}$ & $1,08 \mathrm{~b}$ & $4,50 \mathrm{a}$ \\
90 & $24,51 \mathrm{a}$ & $26,07 \mathrm{a}$ & $2,20 \mathrm{~b}$ & $14,0 \mathrm{a}$ \\
\hline
\end{tabular}

${ }^{1 / A s}$ médias foram comparadas pelo teste $t$ a $5 \%$, considerando amostras com variâncias equivalentes para CE com pesos de $30 \mathrm{~g}$ e $90 \mathrm{~g}$ e PI com peso de $10 \mathrm{~g}$, e amostras presumindo variâncias diferentes para CE com peso de $10 \mathrm{~g} \mathrm{e} \mathrm{PI} \mathrm{com} \mathrm{pesos} \mathrm{de} 30 \mathrm{~g} \mathrm{e} 90 \mathrm{~g}$. As médias seguidas pela mesma letra, para cada característica, em cada linha, não diferem estatisticamente.

\section{CONCLUSÕES}

A pipoqueira de ar quente é um sistema eficiente de avaliação de CE em programas de melhoramento.

Neste equipamento podem ser empregados 10 $\mathrm{g}$ na avaliação de plantas e até $90 \mathrm{~g}$ para avaliações de famílias.

O aparelho de microondas é também um sistema eficiente de avaliação de CE de plantas e famílias, equivalente à pipoqueira de ar quente, podendo, também, ser utilizado em programas de melhoramento.

Para avaliação da CE no aparelho de microondas, pode-se empregar saco de papel pardo comum.

Para avaliação de plantas, podem ser empregados 10 gramas e 140 segundos. Para a avaliação de progênies podem ser empregados 30 a 90 gramas de grãos, com o tempo de 220 segundos sob potência máxima.

\section{REFERÊNCIAS BIBLIOGRÁFICAS}

ANDRADE, R.A. Cruzamentos dialélicos entre seis variedades de milho pipoca (Zea mays L.). Viçosa, 1996. 79p. Dissetação (M.S.) - Universidade Federal de Viçosa.

BRUNSON, A.M. Popcorn selection for added popping expansion would pay large growers. Yearbook of Agriculture, v.16, p.441-443, 1931.

DOFING, S.M.; THOMAS-COMPTON, M.A.; BUCK, J.S. Genotype x popping method interaction for expansion volume in popcorn. Crop Science, v.30, p.62-65, 1990.

GREEN JR., V.E.; HARRIS JR., E.D. Popcorn quality and the measurement of popping expansion. Proceedings of The Soil and Crop Science Society of Florida, v.20, p.28-41, 1960.

MATTA, F.P.; VIANA, J.M.S. Heterose e conseqüências de endogamia em populações de milho pipoca (Zea mays L.). In: SIMPÓSIO DE INICIAÇÃO CIENTÍFICA, 6., Viçosa, 1996. Resumos. Viçosa: UFV, 1996. p.33.

METZGER, D.D.; HSU, K.H.; ZIEGLER, K.E.; BERN, C.J. Effect of moisture content on popcorn popping volume for oil and hot-air popping. Cereal Chemistry, v.66, p.247-248, 1989.

MOHAMED, A.A.; ASHMAN, R.B.; KIRLEIS, A.W. Pericarp thickness and other kernel physical characteristics relate to microwave popping quality of popcorn. Journal of Food Science, v.58, p.342-346, 1993.

PACHECO, C.A.P.; CASTOLDI, F.L.; ALVARENGA, E.M. Efeito do dano mecânico na qualidade fisiológica e na capacidade de expansão de sementes de milho pipoca. Revista Brasileira de Sementes, v.18, p.267-270, 1996.

ROSHDY, T.H.; HAYAKAWA, K.; DAUN, H. Time and temperature parameters of corn popping. Journal of Food Science, v.49, p.1412-1418, 1984.

SONG, A.; ECKHOFF, S.R.; PAULSEN, M.; LITCHFIELD, J.B. Effects of kernel size and genotype on popcorn popping volume and number of unpopped kernels. Cereal Chemistry, v.68, p.464-466, 1991.

Recebido em 06.09.00 